\title{
EXPLAINING THE BENEFITS OF TEAM- GOALS TO SUPPORT COLLABORATION
}

\author{
Annett Schöttle ${ }^{1}$ and Patrícia A. Tillmann ${ }^{2}$
}

\begin{abstract}
The importance of a collaborative environment to achieve success in projects has been widely discussed in the literature and different mechanisms have been developed and introduced to support a collaborative approach to construction projects, i.e. new forms of agreement, new office arrangements, financial incentives, a shared risk and reward approach, the development of shared goals, etc. However, the literature related to these mechanisms is predominantly prescriptive, with little evidence and justification on why some of these mechanisms might be important to support collaboration. In this paper, we focus on discussing the development of shared goals as a means to support collaboration. We collected findings from two case studies in which an explicit process for goal setting and tracking was used to emphasize a collaborative environment. The technique used in these projects are not new and have been documented elsewhere. However, the benefits of these kind of techniques to support collaboration have not been fully explored in the lean construction community. Thus, the intent of this paper is to report some of the benefits that a goal setting exercise brought to two construction projects while having a theoretical discussion to explain why such process can be beneficial and should also be considered - along with other mechanisms - as an important element to support collaboration.
\end{abstract}

\section{KEYWORDS}

Collaboration, commitment, goals-setting, process, vision

\section{INTRODUCTION}

Collaboration can be understood as an "interorganizational relationship with a common vision to create a common project organization with a commonly defined structure and a new and jointly developed project culture, based on trust and transparency; with the goal to jointly maximize the value for the customer by solving problems mutually through

1 PhD Candidate at Karlsruhe Institute of Technology (KIT), Institute for Technology and Management in Construction (TMB), Advisor: Prof. Dr.-Ing. Fritz Gehbauer, M.S.\& Senior Consultant at Refine Projects AG, Stuttgart, Germany, +4915156561529, annett.schoettle@protonmail.com

2 Senior Lean Manager, University of California San Francisco, California, +1415-279-9102, patricia.andretillmann@ucsf.edu 
interactive processes, which are planned together, and by sharing responsibilities, risk, and rewards among the key participants" (Schöttle et al 2014).

Past research advocates that to support collaboration, teams should share a common vision and define shared goals (Appley and Winder 1977, Mattessich and Monsey 1992, Schrage 1995). Past studies within the IGLC community have demonstrated that continuous improvement efforts that include an explicit goal-setting and progress tracking process can support the establishment of team priorities, enable candid conversations and collective problem solving, as well as improve interpersonal relationships (see Tillmann 2014a, 2014b). However, those studies do not explicitly highlight the contributions of a goal setting and tracking process for collaboration from a theoretical standpoint.

Thus, the aim of this research was to understand the theoretical contributions of an explicit process for goal setting and progress tracking to support collaboration. To answer this question, a literature review was carried out to explain the theory of collaboration. Then a framework with the key characteristics of collaboration was developed to support the analysis of empirical data. Two case studies that used a similar process described in Tillmann 2014a and 2014b were analyzed. Findings are presented in this paper with the accompanying theoretical discussion.

\section{LITERATURE REVIEW}

\section{COLLABORATION AND GOALS}

Schöttle et al. (2014) analyzed the literature between 1977 to 2014 to understand the difference between cooperation and collaboration and concluded that cooperation and collaboration can be described as a continuum where cooperation is on end and real collaboration on the other end. Between both ends different concepts exists, which weren't further detailed. Their conclusion relies on the Wood and Gray (1991) definition that collaboration requires "shared rules, norms, and structures, to act or decide on issues" and on the approach of Denise (1999), Sioutis and Tweedale (2006), CamarinhaMatos et al. (2009), and Podean et al. (2011) that collaboration is a "shared creation." Besides, collaborations are temporary (e. g. Schrage 1995, Kumar and van Dissel 1996, Denise 1999, Sioutis and Tweedale 2006, Garmann Johnsen and Ennals 2012), because if the purpose is fulfilled the collaboration ends (Schöttle et al 2014), and fragile, because of the development process which usually contain complex dependencies (Wood and Gray 1991, Kumar and van Dissel 1996, Thomson and Perry 2006, and Huxham 2006). Thus, collaboration "emerges over time, while actors interact formally and informally with each other to create new rules and structures" (Schöttle et al. 2014 based on Thomson and Perry 2006, and Thomson et al. 2009). Therefore, to develop collaboration many authors argue that common goals are required (Appley and Winder 1977, Mattessich and Monsey 1992, Schrage 1995, Kahn 1996, Huxham and Vange 2000, Huxham 2006, Camarinha-Matos and Abreu 2007, Camarinha-Matos et al. 2009, and Garmann Johnsen and Ennals 2012). For example, Mattessich and Monsey (1992) state that beside others "concrete attainable goals and objectives" as well as a "shared vision" 
are success factors of collaboration. Also, Schrage (1995) names a shared understood goal as a success factor for collaboration.

Nevertheless, because Schöttle et al. (2014) point out that the terms cooperation and collaboration are used interchangeable, the purpose of this paper is not to make a distinction between these two terms nor will this paper define the degree of cooperation or collaboration the teams of the two cases achieved. In accordance to Schöttle et al. (2014), we argue that full collaboration is the perfection of working together; a target which teams strive to achieve, but never totally achieve. Therefore, the object of this paper is to justify the use of tools which help to develop collaboration

\section{GOALS, GOAL-SETTING, AND GOAL COMMITMENT}

Widmeyer and Ducharme (1997) define goals as "guides for action." Johnson and Johnson (2009) agree with Widmeyer and Ducharme (1997) and explain that beside that, goals are important, because they motivate behavior, provide the basis for conflict resolution, and prerequisite for assessment and evaluation. Latham and Locke (2006) define goals as "a level of performance proficiency that we wish to attain, usually within a specific time period." Furthermore, they state that goals regulate behaviors, increase efforts, encourage the search for strategies, and give a meaning to a task as well as accomplishment and therefore goal-setting gives direction and affect action (Latham and Locke 2006) and specify goal-setting as a "discrepancy-creating process, in that the goal creates constructive discontent with our present performance" (Latham and Locke 2006). Latham and Yukl (1975) define goal-setting as a mean to improve performance. Then again, setting goals is based on importance and self-efficacy (Locke and Latham 2002, Latham and Locke 2006) and affected by leadership (Locke and Latham 2006).

By reviewing the literature of goal-setting theory Locke (1996) argue that the more difficult and specific goals are the more critical is the commitment for achieving the goal, but the higher is the performance if the commitment is there. The commitment in turn depends on the importance and the attainability of the goal for the individual commitment giver as well as the self-efficacy to accept feedback that helps to set and perform goals (Locke 1996). Therefore, Locke (1996) defines goal commitment as "the degree to which the person is genuinely attached to and determined to reach the goals." This in turn depends on whether or not goals are assigned or self-set. Hinsz (1995) summarize that goal commitment is assured if individuals feel somehow involved in the selection of assigned goals or if it is ensured that self-set goals are specific and challenging and e. $\mathrm{g}$. Locke (1996) and Locke and Latham (2006) explain that assigning goals without explanation result in less commitment. Thus, goal commitment is important link goal and behavior (Klein et al. 2001). Besides, when implementing a goal setting and tracking exercise it needs to be considered that the process is influenced by past experience and affected by direction, persistence, effort, and training to not fall back to old habits (Locke 1996).

\section{GROUP-GOAL-SETTING AND EVALUATION}

Johnson and Johnson (1987) define a group goal as "a future state of affairs desired by enough members of a group to motivate the group to work towards its achievement." 
Zander (1971) explains that although an agreement among the group exists, team goals consist of four types of goals (1) group goals, (2) group goals for members, (3) members goals for self, and (4) members goals for group, and that those individual and group goals are in a circular relation to each other. Team goals help the group to stay focused (Johnson and Johnson 1987), can increase team cohesion (Johnson and Johnson 1987, Widmeyer and Ducharme 1997) and team effectiveness (Widmeyer and Ducharme 1997). To achieve commitment regarding group goals Johnson and Johnson (2009) identified two ways: (1) goals have to be specific, trackable and measurable, achievable and challenging, relevant to the members, and transferable to other situations, or (2) goals need to be formed together by the group. Besides, the desirability of a goal and the relation of the team impact the commitment (Johnson and Johnson 2009). Pritchard et al. (1988) argue that systems which are developed by their users are much more effective as systems which were imposed top-down, because involved participants are much more aware about their issues and needs to design a functional process. Durham et al. (1997) suggest that "teams engaged in new, complex tasks should be allowed to set team goals, even if formal goals are assigned. Team-set goals are more likely to lead to effective performance, because they take into account what teams believe they can achieve." Furthermore, Seijts and Latham (2000) found that the larger the group the more important it is (1) to align individual and group goals, (2) fostering the collective beliefs of efficacy, (3) to increase the belief that collaboration results in positive outcomes, and (4) to develop commitment to group goals to avoid the occurrence of social dilemma.

\section{RESEARCH METHOD}

A case study approach was chosen for this research as it allows for a deep understanding of the subject (Yin, 2014). The aim of the case studies was to collect empirical data that could support the evaluation of a goal-setting mechanism and its contribution to the different elements that constitute a collaborative environment. Two case studies were carried out. Both cases had limited ability to introduce other mechanisms to support collaboration (i.e. multi-party agreements, shared financial structure). However, in both cases, a goal setting and tracking exercise was utilized.

In order to support data collection and analysis, an analytical framework was developed based on the reviewed literature (Table 1). Data was mainly collected through interviews. The interviews were carried out in November $2014^{3}$. Participants of the interviews were the owner, general contractor, and the architect in case 1 and project manager and general contractor in case 2. Open-ended questions were asked regarding the project delivery system, implemented methods and tools as well as about the working environment. Afterwards the interviews were transcript and based on Mayring (2010) and Kuckartz (2014) qualitative content analysis used to analyze the interviews.

3 The interviews were collected, transcript, codified, and analyzed by the first author as part of her $\mathrm{PhD}$ thesis. 
Table 1: Observed collaboration characteristics

\begin{tabular}{lcc}
\hline Observed characteristics & Case 1 & Case 2 \\
\hline Inter-organizational relationship & $\mathrm{x}$ & $\mathrm{x}$ \\
Common vision & $\mathrm{x}$ & $\mathrm{x}$ \\
Common project organization & $\mathrm{x}$ & $\mathrm{x}$ \\
Commonly defined structure & $\mathrm{x}$ & $\mathrm{x}$ \\
Jointly developed project culture & $\mathrm{x}$ & $\mathrm{x}$ \\
Trust & $\mathrm{x}$ & $\mathrm{x}$ \\
Transparency & $\mathrm{x}$ & $\mathrm{x}$ \\
Jointly maximize customer value & $\mathrm{x}$ & $\mathrm{x}$ \\
Interactive processes, which are planned together & $\mathrm{x}$ & $\mathrm{x}$ \\
Sharing responsibilities & $\mathrm{x}$ & $\mathrm{x}$ \\
Sharing risk and rewards & $\mathrm{x}$ & $\mathrm{x}$ \\
\hline
\end{tabular}

\section{CASE STUDIES DESCRIPTION AND FINDINGS}

The two case studies in which a goal-setting and tracking exercise was carried out were both Laboratory renovations. The first project was the renovation of an anatomy teaching lab on the $13^{\text {th }}$ floor of an occupied building (US\$ 7.4M, substantial completion in August 2012). The second case was a project to remediate the existing plumbing and electrical system underneath the first floor (US\$ $21.4 \mathrm{M}$, substantial completion June 2014). In both cases, a traditional construction management at risk contract was used, with no arrangements to share risks and rewards, no multi-party agreement and no colocation.

On both public projects, different tools and techniques were implemented by the general contractor (which was the same in both projects). Those include: Last Planner System, Building Information Modelling and a shared goal-setting and tracking exercise to support collaboration. The participants of the self-setting goal and evaluation process are summarized in Table 2.

Table 2: Participants of the goal-setting and tracking process

\begin{tabular}{lcc}
\hline Participants & Case 1 & Case 2 \\
\hline General contractor & $\mathrm{x}$ & $\mathrm{x}$ \\
Owner and facilities maintenance & $\mathrm{x}$ & $\mathrm{x}$ \\
Project manager (external consultant owner) & $\mathrm{x}$ & $\mathrm{x}$ \\
Architect & $\mathrm{x}$ & - \\
Design team & $\mathrm{x}$ & $\mathrm{x}$ \\
Main subcontractors & - & $\mathrm{x}$ \\
User representative & $\mathrm{x}$ & - \\
\hline
\end{tabular}


The process included a series of workshops to develop an agreed upon vision and a periodic review of progress towards the goals, in which project participants would discuss current status and opportunities. Figure 1 shows the PDCA-cycle of the implemented process. Further explanation can be found in Tillmann (2014a, 2014b).

The vision established by the team reflected specific goals that needed to be achieved. These included project goals such as to be on time and on schedule and to incorporate built in quality. Besides the typical project goals, the team set specific goals to support the working environment. For example, one goal was "having fun".

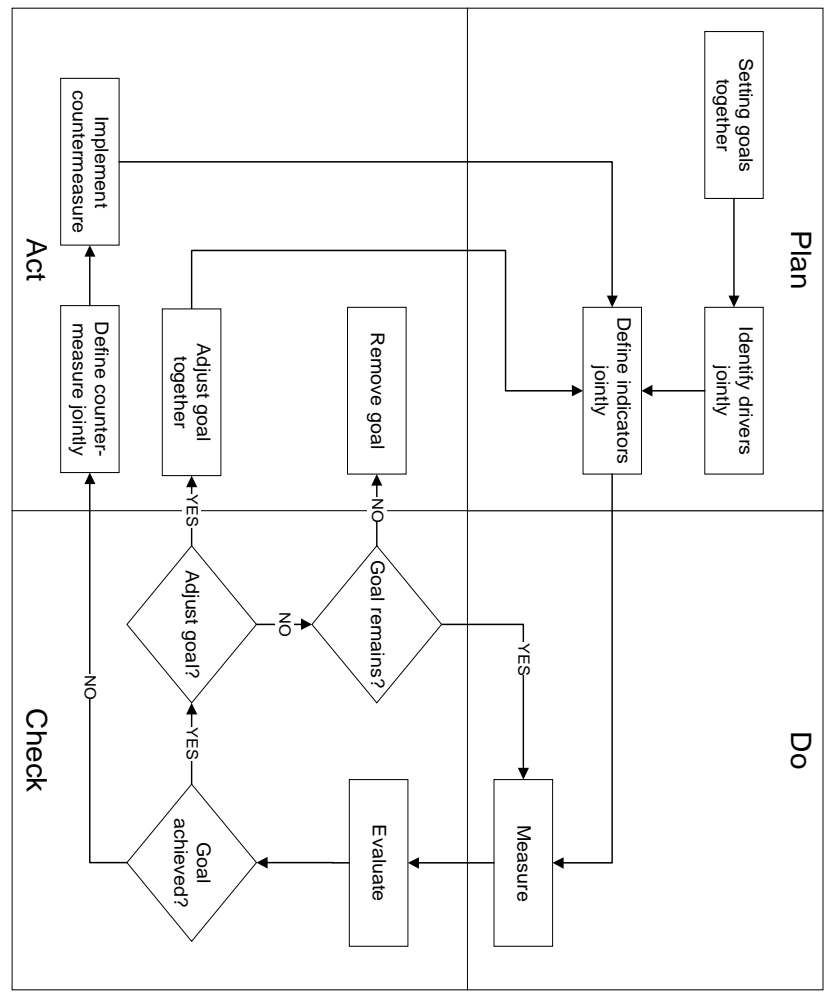

Figure 1: PDCA-cycle of goal-setting and tracking process

To track progress, team used both quantitative (i.e. expected vs. actual costs, expected vs. actual schedule performance) and qualitative data. Qualitative data was gathered through surveys and focused on the opinion of team members, especially regarding soft aspects of project management (i.e. teamwork, fun, building relationships, etc.). In case that there was a discrepancy between goal and progress, countermeasures were defined and implemented. If the team evaluated that "having fun" was not achieved one countermeasure would be to have lunch together and establish a rule to not talk about work during lunch time, for instance. Instead, people would talk about their private life, which contributed for increased empathy and stronger relationships.

Interviewees mentioned that some progress measurements should have been defined more clearly, and that goals such as building new relationships needed to be adjusted, because they change over time. Figure 1 shows how goal adjustment should be included 
as part of the process. Furthermore, it was stated that the individual evaluation of goals which were not self-set were difficult. This aligns with the statement of Locke and Latham (2002) and Latham and Locke (2006), who argue that goals need to be relevant for the individual. Thus, if a lack of identification occurs, the measurement needs to be clearly defined and might be adjusted in terms of group evaluation than by every individual. Despite these opportunities for improvement, project team members highlighted major benefits of this process for enhancing collaboration. These included:

- Open and increased communication among team members;

- Aligned expectations and improved team focus;

- Possibility to communicate dissatisfaction, talk about frustration and address issues;

- Smoother workflow;

- Development of an understanding of different personalities, while building relationships;

- Developing collaboration and building Strengthened trust; and

- Influenced the working environment and improved satisfaction.

\section{DISCUSSION}

Even though the characteristics of these case studies differed from the projects analyzed by Tillmann (2014a, 2014b), i.e. no integrated agreement, no arrangement to share risks and rewards, positive results were found. Teams observed improved relationships, greater empathy, an enhanced ability to candidly talk about problems and engage in problemsolving to support continuous improvement. This study provides further evidence that support the findings of other authors. As different studies point out, a goal-setting and tracking exercise which is carried out by project teams themselves increased communication and coordination (Johnson and Johnson 2009) and served as a "guide for action" (Widmeyer and Ducharme 1997). Furthermore, setting goals motivated the team (Widmeyer and Ducharme 1997, Locke and Latham 2006) and provided the basis for conflict resolution (Widmeyer and Ducharme 1997).

Table 3 gives an overview of collaboration characteristics based on the definition of Schöttle et al. (2014). The authors evaluate the table, based on their observation and on the interviews. It can be seen that the goal-setting and tracking process impacted collaboration, despite the fact that not all supporting mechanisms were in place, i.e. multi-party agreements or shared risks and rewards. Therefore, the goal-setting and tracking exercises were critically important for helping to align the different parties with respect to 7 out of 11 mechanisms that are considered desirable for supporting collaboration.

In the process of agreeing on goals, expectations were clarified, and the team got a better understanding of each other's perspective. Moreover, targets were defined bottomup, so that the different team members identified themselves within the goals (as previously observed by Locke (1996). Setting and tracking also social goals helped the team members to pay more attention to issues regarding the working environment and 
define countermeasure for team building on time. Overall, the goal measurement helped to see if things were going in the right direction or if the team needed to change something to be on track. Thus, in accordance to the literature review, the collaboration among the team members developed over time (Schöttle et al. 2014, Thomson and Perry 2006, Thomson et al. 2009), because they shared goals, informally interact with each other, opened up the discussion about what was important for their performance and based on that discussion, developed rules and norms to proceed in the right path.

Table 3: Affected collaboration characteristics

\begin{tabular}{lcc}
\hline Affected characteristics & Case 1 & Case 2 \\
\hline Inter organizational relationship & Yes & Yes \\
Common vision & Yes & Yes \\
Common project organization & No & No \\
Commonly defined structure & No & No \\
Jointly developed project culture & Yes & Yes \\
Trust & Yes & Yes \\
Transparency & Yes & Yes \\
Jointly maximize customer value & Yes & Yes \\
Interactive processes, which are planned together & Yes & Yes \\
Sharing responsibilities & No & No \\
Sharing risk and rewards & No & No \\
\hline
\end{tabular}

\section{CONCLUSION}

This paper provides greater insight into what collaboration means from a theoretical standpoint and greater support on understanding why goal setting exercises are important. Furthermore, it provides evidence that support past studies and reinforces the understanding of the contribution of establishing common goals in construction projects, even when companies are not aligned commercially. A goal-setting and tracking processes makes explicit important issues which are usually not verbalized by project teams. By clarifying goals and defining actions jointly, individuals focus on problem solving and achieve a greater alignment among themselves. Thus, the effect of the goalsetting and tracking process described here with respect to collaboration is high, even in traditional project arrangements. Some limitations include: the research did not consider the role of leadership during the team goal-setting and tracking exercises, and it did not differentiate between assigned, participative, and self-set goals. Therefore, additional research about goal-setting and tracking to understand and improve the process and to enhance collaboration is necessary. 


\section{ACKNOWLEDGMENT}

The authors want to thank the German Academic Exchange Service (DAAD - Deutscher Akademischer Austausch Dienst), the Institute of Technology and Management in Construction at Karlsruhe Institute of Technology (KIT), the Product Production System Laboratory (P2SL) at UC Berkeley and the UCSF Capital Program Department (REPCAPS) for supporting this research.

\section{REFERENCES}

Appley, D.G. and Winder, A.E. (1977). "An Evolving Definition of Collaboration and Some Implications for the World of Work." J. of App. Behav. Sci., 13(3), 279-291.

Camarinha-Matos, L.M., and Abreu, A. (2007). "Performance indicators for collaborative networks based on collaboration benefits." Production Planning \& Control: The Management of Operations, 18(7), 592-609.

Camarinha-Matos, L.M., Afsarmanesh, H., Galeano, N. and Molina, A. (2009). "Collaborative networked organizations - Concepts and practice in manufacturing enterprises." Computers \& Industrial Engineering, 57(1), 46-60.

Denise, L. (1999). „Collaboration vs. C-three (Cooperation, Coordination, and Communication)." INNOVATING, 7(3).

Garmann Johnsen, H.C., and Ennals, R. (2012). "Introduction: Collaborative Advantage in Regional Economies.” In: H.C Johnsen Garmann and R. Ennals, ed. (2012). Creating Collaborative Advantage: Innovation and Knowledge Creation in Regional Economie.

Hinsz, V.B. (1995). Goal Setting by Groups Performing an Additive Task. Journal of Applied Social Psychology, 25(11), 965-990.

Huxham, C., and Vange, S. (2000). "Ambiguity, Complexity and Dynamics in the Membership of Collaboration." Human Relations, 53(6), 771-806.

Huxham, C. (2006). "Theorizing collaboration practice.” Public Mgmt. Review, 5(3), 401423.

Johnson, D.W., and Johnson, F.P. (2009). Joining together: Group theory and group skills. 10. ed., Pearson, Upper Saddle River, NJ [u.a.].

Kahn, K.B. (1996). "Interdepartmental integration: A definition with implications for product development performance." J. of Prod. Innov. Mgmt., 13(2), 137-151.

Klein, H.J., Wesson, M.J., Hollenbeck, J.R., Wright, P.M., and DeShon, R.P. (2001). "The Assessment of Goal Commitment: A Measurement Model Meta-Analysis." Organizational Behavior and Human Decision Processes. 85(1), 32-55.

Kuckartz, U. (2014). Qualitative Inhaltsanalyse. Methoden, Praxis, Computerunter-stützung. Beltz Juventa, Weinheim und Basel, 2 edition.

Kumar, K., and van Dissel, H.G. (1996). "Sustainable Collaboration: Managing Conflict and Cooperation in Interorganizational Systems." MIS Quarterly, 20(3), 279-300.

Latham, G. P. and Yukl, G.A. (1975). "A Review of Research on the Application of Goal Setting in Organizations." The Academy of Management Journal, 18(4), 824-845.

Latham G.P and Locke, E.A. (2006). "Enhancing the Benefits and Overcoming the Pitfalls of Goal Setting.” Organizational Dynamics. 35(4), 332-340.

Locke E.A. (1996). "Motivation through conscious goal setting." Applied \& Preventive Psychology, 5, 117-124. 
Locke, E.A. and Latham, G.P. (2002). "Building a practically useful theory of goal setting and task motivation." American Psychologist, 57, 705-717.

Locke, E.A. and Latham G.P (2006). "New Directions in Goal-Setting Theory." Current direction in Goal-Setting Theory. 15(5), 265-268.

Mayring, P. (2010). Qualitative Inhaltsanalyse: Grundlagen und Techniken, Vol. 11. Beltz, Weinheim und Basel.

Mattessich, P.W., and Monsey, B.R. (1992). Collaboration: What makes it work. Saint Paul, MN: Amherst H. Wilder Foundation.

Pritchard, R.D.; Jones, S.D.; Roth, P.L.; Stuebing, K.K.; and Ekeberg, S.E. (1988). Effects of Group Feedback, Goal Setting, and Incentives on Organizational Productivity. Journal of Applied Psychology, 73(2), 337-358.

Podean, M.I., Benta, D., Rusu, L. (2011). "About creativity in collaborative systems - Why it matters and how it can be supported" Proc. of the International Conference on $e$ Business, Seville; Spain, 151-154.

Schöttle, A., Haghsheno, S. andGehbauer, F. (2014). "Defining Cooperation and Collaboration in the Context of Lean Construction." Pro. Of the 23rd Ann. Conf. of the Int'l. Group for Lean Construction (IGLC). Oslo, Norway.

Schrage, M. (1995). No more teams!: Mastering the dynamics of creative collaboration. Currency Doubleday, New York.

Seijts, G.H., and Latham, G.P. (2000). The Effects of Goal Setting and Group Size on Performance in a Social Dilemma. Canadian Journal of Behavioural Science, 32(2), 104116.

Sioutis, C., and Tweedale, J. (2006). "Agent Cooperation and Collaboration." KnowledgeBased Intelligent Information and Engineering Systems: Proc. of the 10th International Conference, Gabrys, B., Howlett, R.J., and Jain, L.C., eds., Springer, Berlin Heidelberg New York, 464-471.

Thomson, A.M., and Perry, J.L. (2006). "Collaboration process: Inside the black box." Public Administration Review, 66, 20-32.

Thomson, A.M., Perry, J.L., and Miller, T.K. (2009). "Conceptualizing and measuring collaboration." J. of Pub. Administr. Research and Theory, 19(1), 23-56.

Tillmann, P., Berghede, K., Ballard, G., and Tommelein, I.D. (2014). "Developing a Production System on IPD: Considerations for a Pluralistic Environment." Pro. Of the 23rd Ann. Conf. of the Int'l. Group for Lean Construction (IGLC). Oslo, Norway.

Tillmann, P., Ballard, G., and Tommelein, I.D. (2014). "A Mentoring Approach to Implement Lean Construction." Pro. Of the 23rd Ann. Conf. of the Int'l. Group for Lean Construction (IGLC). Oslo, Norway.

Tjosvold, D., and Tsao, Y. (1989). "Productive Organizational Collaboration: The Role of Values and Cooperation." J. of Organizational Behavior, 10(2). 189-195.

Widmeyer, W. N., and Ducharme, K. (1997). "Team building through team goal setting." Journal of Applied Sport Psychology, 9(1), 97-113.

Wood, D.J., and Gray, B. (1991). "Toward a Comprehensive Theory of Collaboration" Journal of Applied Behavioral Science, 27(2), 139-162.

Yin, R.K. (2014). Case study research Design and Methods. $5^{\text {th }}$ edition, Saga, Los Angeles, Calif. [u.a.].

Zander, A. (1971). Motives and goals in groups. Academic Press, New York. 